Nisho-99/1

\title{
Axionic Boson Stars in Magnetized Conducting Media
}

\author{
Aiichi Iwazaki \\ Department of Physics, Nishogakusha University, Shonan Ohi Chiba 277, Japan.
}

(January 8, 1999)

\begin{abstract}
Axions are possible candidates of dark matter in the present Universe. They have been argued to form axionic boson stars with small masses $10^{-14} M_{\odot} \sim 10^{-11} M_{\odot}$. Since they possess oscillating electric fields in a magnetic field, they dissipate their energies in magnetized conducting media such as white dwarfs or neutron stars. At the same time the oscillating electric fields generate a monochromatic radiation with energy equal to mass of the axion. We argue that the effect of the energy dissipation can be seen in the old white dwarfs. In particular, We show that colliding with sufficiently cooled white dwarfs, plausible candidates of MACHO, the axionic boson stars dissipate their energies in the dwarfs and heat up the dwarfs. Consequently the white dwarfs in the halo can emit detectable amount of thermal radiations with the collision. On the other hand, the monochromatic radiations can be seen only during the collision; a period of the dwarf passing the axionic boson star. Assuming that MACHO are dark white dwarfs, we show that there is a threshold in luminosity function of the white dwarfs below which the number of the white dwarfs in the halo increase discontinuously. The threshold in the luminosity function is expected to be located around $10^{-5.5} L_{\odot} \sim 10^{-7} L_{\odot}$. Its
\end{abstract}


precise value is determined by the mass of the axionic boson stars dominant in the halo.

\section{INTRODUCTION}

The axion is the Goldstone boson associated with Peccei-Quinn symmetry [1], which was introduced to solve naturally the strong $\mathrm{CP}$ problem. In the early Universe some of the axions condense and form topological objects [2, [3], i.e. strings and domain walls, although they decay below the temperature of QCD phase transition. After their decay, however, they have been shown to leave a magnetic field [4] as well as cold axion gas as relics in the present Universe; the field is a candidate of a primordial magnetic field supposed to lead to galactic magnetic fields observed in the present Universe.

In addition to these topological objects, the existence of axionic boson stars has been argued [5.6]. It have been shown numerically [6] that in the early Universe, axion clumps are formed around the period of $1 \mathrm{GeV}$ owing to both the nonlinearity of an axion potential leading to an attractive force among the axions and the inhomogeneity of coherent axion oscillations. Namely, when the temperature of the Universe decreases and the axion potential is generated by QCD instantons, the inhomogeneity of the coherent axion oscillation on the scale beyond the horizon gives rise to localized clumps due to the attractive force of the potential. These clumps are called axitons since they are similar to solitons in a sense that its energy is localized. Then, the axitons contract gravitationally to axionic boson stars [7,8] after separating out from the cosmological expansion. They are solitons of coherent axions bounded gravitationally. ( The axions are represented with a real scalar field, which has

been shown to possess solutions of oscillating boson stars [8]. ) The masses of the axionic boson stars have been estimated roughly to be order of $\sim 10^{-12} M_{\odot}$. Eventually we expect that in the present Universe, there exist the axionic boson stars as well as the axion gas as dark matter candidates. It has been estimated [9] that a fairly amount of the fraction of the axion dark matter is composed of the axionic boson stars. 
In this paper we wish to point out an intriguing observable effect associated with the coherent axionic boson stars; we call them axion stars. Namely, they dissipate their energies in magnetized conducting media such as magnetic white dwarfs or neutron stars so that the temperature of the media increases and thermal radiations are emitted. The phenomena are caused by oscillating electric fields generated by the axion field under external magnetic fields; their frequency is given by the mass of the axion. The electric fields induce electric currents in the conducting media and loose their energies owing to the existence of electric resistances. Consequently the axion stars dissipate their energies in the magnetized conducting media. Although the electric fields themselves are small, the total amount of the energy dissipation is very large because the dissipation arises all over the volume of the axion stars or the volume of the magnetized conducting media: Radii of the white dwarfs ( the neutron stars ) are typically $10^{9} \mathrm{~cm}\left(10^{6} \mathrm{~cm}\right)$, while radii of the axion stars of our concern are such as $10^{6} \mathrm{~cm} \sim 10^{10} \mathrm{~cm}$. Consequently detectable amount of radiations are expected from the media heated in this way. In particular we are concerned with old white dwarfs which are plausible candidates of MACHO [10] and have been cooled sufficiently; they are dark enough to be invisible. Thus the effect of the energy dissipation of the axion star in such white dwarfs possessing small specific heats is so large that the luminosity of the dwarfs after the collisions with the axion stars increases so as for the dwarfs to be observable.

In particular, we point out that there is a threshold in luminosity function of the halo white dwarfs ( luminosity function describes, roughly speaking, the number density of the white dwarfs as a function of luminosity ). Namely the luminosity function increases discontinuously below the threshold of a certain luminosity. To explain it, we first point out that the white dwarfs in the halo are sufficiently old so that their luminosity is quite low to be invisible. Especially, according to a recent cooling model, the white dwarfs with helium rich atmosphere are expected to have been cooled so that their luminosity is less than $10^{-7} L_{\odot}$ at their age $\sim 1.2 \times 10^{10}$ years. Here we assume that MACHO are such white dwarfs with helium rich atmosphere. Thus they are invisible with present observational apparatus unless they are located quite near the sun. Then since they have few internal thermal energies, 
their temperatures increase dramatically with the dissipation of the energy of the axion star in the white dwarfs. We show that the luminosity gained by the white dwarfs with the collision is about $10^{-5.5} L_{\odot} \sim 10^{-7} L_{\odot}$ depending on the mass of the axion star; the mass of our concern is $10^{-11} M_{\odot} \sim 10^{-14} M_{\odot}\left(L_{\odot}\right.$ denotes the luminosity of the sun $)$. Therefore, this luminosity is expected to be the threshold luminosity of the white dwarf luminosity function in the halo.

In addition to these thermal radiations from the magnetized medium, the oscillating electric fields associated with the axion star in the magnetized medium generate monochromatic radiations with energy equal to the mass of the axion. Since the electric fields arise only during the axion star being exposed to an external magnetic field of the magnetized media, the radiations are emitted only in a period of the media passing the axion star. Therefore, we expect that we first observe the monochromatic radiations in a short period and then observe the thermal radiations which are emitted until the dissipation energy deposited by the axion star is exhausted. This thermal radiations are observed possibly as a nova which is an old white dwarf heated with the collision. Here we should mention that since the rate of the collision between the white dwarfs and the axion stars is much small, it seems difficult that we observe the monochromatic radiations.

Because the strength of the electric fields is proportional to the strength of the magnetic field, the phenomena are distinctive of the strongly magnetized media. We show that the amount of the energy dissipated in white dwarfs, for instance, with mass $\sim 0.5 M_{\odot}$ and with magnetic field larger than $10^{5}$ Gauss is approximately given by $10^{42} \operatorname{erg} M_{a} / 10^{-12} M_{\odot}$ where $M_{a}\left(M_{\odot}\right)$ is the mass of the axion star ( the sun ). Namely in almost of all cases, whole energy of the axion star is dissipated in such white dwarfs. In our discussions we neglect, for simplicity, gravitational effects of the magnetized conducting media on the axion stars when they collide with the media. Later we discuss on this point.

In section (2) we present our solutions of the axionic boson stars with small masses. The axion field of the solutions oscillates with the frequency of the axion mass. We show explicitly relations between the mass and the radius of the axion star, which are exploited 
for the estimation of the amount of the energy dissipation in magnetized conducting media. In section (3) we describe an intriguing phenomenon that the axionic boson star induces an oscillating electric field when it is exposed to an external magnetic field. This electric field induces electric currents carried by ordinary charged particles in the magnetized conducting media. Thus the axion stars dissipate their energies in such media. The axion star in the magnetic field possesses another oscillating electric current even in nonconducting media such as vacuum owing to an interaction of the axion field with the electromagnetic fields. The current [11] is composed of the axion field although the axions are neutral. Owing to these two types of the oscillating currents, the axion stars dissipate their energies in the conducting media. Thus the media are heated so that thermal radiations are emitted. The application of these phenomena is discussed in section (4) and section (5) where white dwarfs and neutron stars are discussed as magnetized conducting media, respectively. separately. We summarize our results in the final section (6).

\section{AXIONIC BOSON STAR}

Let us first explain our solutions of the axionic boson stars. Originally Seidel and Suen 8] have found solutions of a real scalar axion field, $a$, coupled with gravity. Their solutions represent spherical oscillating axion stars with masses of the order of $10^{-5} M_{\odot}$; the solution possesses oscillation modes with various frequencies. ( Static regular solutions have been shown not to exist in the massless real scalar field coupled with gravity. Even in the massive real scalar field such solutions have not yet been obtained. ) Although these axion stars are oscillating, they are stable solitons composed of the axions coupled with gravity; they are similar to the "breather" solution of the $(1+1)$-dimensional sine-Gordon model. Axion

stars of our concern, on the other hand, are ones with much smaller masses, $\sim 10^{-14} M_{\odot}$. This is because according to arguments of Kolb and Tkachev [6,7, 9, the axion stars produced in early Universe have masses typically such as $10^{-12} M_{\odot} \Omega_{a} h^{2}$ where $\Omega_{a}$ is the ratio of the axion energy density to the critical density in the Universe and $h$ is Hubble constant in 
the unit of $100 \mathrm{~km} \mathrm{~s}^{-1} \mathrm{Mpc}^{-1}$. They have been produced after the period of QCD phase transition with axions contracting due to both effects of the gravitational attraction and the attraction of the axion potential.

So, in order to find such solutions and to obtain explicit relations among the parameters, e.g. radius, $R_{a}$, mass, $M_{a}$, e.t.c., of these axion stars, we have numerically obtained solutions of the spherical axionic boson stars [12, 8, 13] in a limit of a weak gravitational field. Relevant equations are a free field equation of the axion and Einstein equations,

$$
\begin{aligned}
\ddot{a} & =\frac{\left(\dot{h_{t}}-\dot{h_{r}}\right) \dot{a}}{2}+a^{\prime \prime}+\left(\frac{2}{r}+\frac{h_{t}^{\prime}-h_{r}^{\prime}}{2}\right) a^{\prime}-m_{a}^{2} a, \\
h_{t}^{\prime} & =\frac{h_{r}}{r}+4 \pi G r\left(a^{\prime 2}-m_{a}^{2} a^{2}+\dot{a}^{2}\right), \\
h_{r}^{\prime} & =-\frac{h_{r}}{r}+4 \pi G r\left(a^{\prime 2}+m_{a}^{2} a^{2}+\dot{a}^{2}\right),
\end{aligned}
$$

where we have assumed gravity being small, i.e. $h_{t, r} \ll 1$ so that the metric is such that $d s^{2}=\left(1+h_{t}\right) d t^{2}-\left(1+h_{r}\right) d r^{2}-r^{2}\left(d \theta^{2}+\sin ^{2} \theta d \phi^{2}\right) ; r, \theta$, and $\phi$ denote the polar coordinates. The first equation is the equation of the axion field $a$. The second and the third equations are Einstein equations. A dot ( dash ) indicates a derivative in time $t(r) . G\left(m_{a}\right)$ is the graviational constant ( the mass of the axion ). The potential term $\sim \sin (a)$ of the axion has been neglected because an amplitude of the field $a$ is sufficiently small for nonlinearity not to arise since the mass of the axion star is small enough. Actually the masses we are concerned with are such as $\sim 10^{-14} M_{\odot}$, while the nonlinearity has been found numerically to arise only for the axion stars with masses larger than $\sim 10^{-9} M_{\odot}$. We need to impose a boundary condition such as $h_{r}(r=0)=0$ for the regularity of the space-time.

Changing the scales such that $\tau=m_{a} t, x=m_{a} r$ and $b=a / m_{a}$, we rewrite the equations as follows,

$$
\begin{aligned}
\ddot{b} & =\dot{V} \dot{b}+b^{\prime \prime}+\left(\frac{2}{x}+V^{\prime}\right) b^{\prime}-b, \\
V^{\prime} & \equiv \frac{h_{t}^{\prime}-h_{r}^{\prime}}{2}=\epsilon\left(\frac{\int_{0}^{x} d x x^{2}\left(b^{\prime 2}+\dot{b}^{2}+b^{2}\right)}{x^{2}}-x b^{2}\right)
\end{aligned}
$$

with $\epsilon=4 \pi G m_{a}^{2}$, where we have expressed $V^{\prime}$ in terms of the field $b$, solving eq(21) and eq(3)); here a dot ( dash ) denote a derivative in $\tau(x)$. We understand that if the gravitational 
effect is neglected ( $\epsilon=0$ ), the equation of $b$ is reduced to a Klein-Gordon equation. Thus the frequency $\omega$ of the field $b$ receives a small gravitational effect of the order of $\epsilon$; $\omega=1-o(\epsilon)$.

We look for such a solution [8] that

$$
b=A_{0} B(x) \sin \omega \tau+o(\epsilon) \sin 3 \omega \tau,
$$

where $B(x)$ represents coherent axions bounded gravitationally with its spatial extension representing the radius of the axion star; $B(x)$ is normalized such as $B(x=0)=1$. Later we find that $A_{0}$ is a free parameter determining a mass or a radius of the axion star. The second term is a small correction of the order of $\epsilon$. Here we comment that previous solutions [8 representing axion stars with larger masses possess more oscillating terms such as $\sin (2 n-1) \omega$ with $n=1,2,$, . Inserting the formula eq(何) into eq(牙) and eq(5) and taking account of the gravitational effects only with the order of $\epsilon$, we find that

$$
k^{2} B=B^{\prime \prime}+\left(\frac{2}{x}+\epsilon A_{0}^{2}\left(T+\frac{3 U^{\prime}}{4}\right)\right) B^{\prime}+\frac{\epsilon A_{0}^{2}(U+v) B}{2}
$$

with

$$
T \equiv \frac{\int_{0}^{x} z^{2} B^{2} d z}{x^{2}} \quad \text { and } \quad U \equiv \int_{0}^{x} d y\left(\frac{\int_{0}^{y} z^{2} B^{\prime 2} d z}{y^{2}}-y B^{2}\right)
$$

where $k^{2}\left(=1-\omega^{2}\right)$ is a binding energy of axions. We have imposed a boundary condition for the consistency such that $V(x=0)=h_{t}(x=0) / 2=\epsilon A_{0}^{2} v \omega \sin 2 \omega \tau$; this is the definition of constant $v$ in the above formula.

We can see that the parameter $\epsilon A_{0}^{2}$ can take an arbitrary value and that it represents the gravitational effect of this system. Namely the mass of the axionic boson star is determined by choosing a value of the parameter. Note that the normalization of $B$ has been fixed in eq(7) although the equation is a linear in $B$.

We may take the value of $v$ without loss of generality such that $v=-U(x=\infty)$. Then the inverse $k^{-1}$ of the binding energy is turned out to represent a radius of the axion star; $B$ decays exponentially such as $\exp (-k x)$ for $x \rightarrow \infty$. It turns out from eq(17) that the choice 
of small values of $\epsilon A_{0}^{2}$ lead to solutions representing the axion stars with small masses and large radii $k^{-1}$.

Before solving eq(17) numerically, it is interesting to rewrite the equation as following. That is, we rewrite the equation by taking only dominant terms of a "potential", $V_{b}$, in $\mathrm{eq}(7)$

$$
V_{b}=\epsilon A_{0}^{2}\left(\left(T+\frac{3 U^{\prime}}{4}\right) B^{\prime}+\frac{(U+v) B}{2}\right)
$$

in the limit of the large length scale; setting $x=\lambda y$, we take a dominant term as $\lambda \rightarrow \infty$. This corresponds to taking the axion stars with their spatial extension being large.

Then, since the dominant term in the limit is the last term in eq(9), $U+v \sim \int_{x}^{\infty} d x x B^{2}$, we obtain the following equation,

$$
\bar{B}=\bar{B}^{\prime \prime}+\frac{2 \bar{B}^{\prime}}{z}+\frac{\bar{B} \int_{z}^{\infty} d y y \bar{B}^{2}}{2}
$$

where we have scaled the variables such that $B^{2}=k^{4} \bar{B}^{2} / \epsilon A_{0}^{2}$ and $x=k^{-1} z$; a dash denotes a derivative in $z$. This equation is much simpler than eq(7), where we need to find each eigenvalue of $k$ for each value of $\epsilon A_{0}^{2}$ given, in order to obtain solutions of the axion stars with various masses. On the other hand, we need only to find an appropriate value of $\bar{B}(z=0)=\epsilon A_{0}^{2} / k^{4}$ in order to obtain such solutions in eq(10). A relevant solution we need to find is the solution without any nodes. Obviously, the solution is characterized by one free parameter, $k^{4}$ or $\epsilon A_{0}^{2}$, which is related to the mass of the axion star. Namely the choice of a value of the mass determines uniquely the properties of the axion star, e.g. radius of the star, distribution of axion field $a$, e.t.c..

Although the equation (10) governs the axion star only with the large radius, we have confirmed by solving numerically original equation (7) that the stars of our concern can be controlled by the equation (10).

We have confirmed that our numerical solutions may be approximated by the explicit formula,

$$
a=f_{P Q} a_{0} \sin \left(m_{a} t\right) \exp \left(-r / R_{a}\right)
$$


where $t(r)$ is time ( radial ) coordinate and $f_{P Q}$ is the decay constant of the axion. The value of $f_{P Q}$ is constrained from cosmological and astrophysical considerations [3],2] such as $10^{10} \mathrm{GeV}<f_{P Q}<10^{12} \mathrm{GeV}$. Corresponding to this constraint, $m_{a}$ is constrained roughly such as $10^{-5} \mathrm{eV}<m_{a}<10^{-3} \mathrm{eV}$.

In the limit of the small mass of the axion star we have found a simple relation [12] between the mass, $M_{a}$ and the radius, $R_{a}$ of the axion star,

$$
M_{a}=6.4 \frac{m_{p l}^{2}}{m_{a}^{2} R_{a}}
$$

with Planck mass $m_{p l}$. Numerically, for example, $R_{a}=1.6 \times 10^{10} m_{5}^{-2} M_{14}^{-1} \mathrm{~cm}$; hereafter we use the notation, $M_{n} \equiv M_{a} / 10^{-n} M_{\odot}$ and $m_{n} \equiv m_{a} / 10^{-n} \mathrm{eV}$. A similar formula has been obtained in the case of boson stars of complex scalar fields [13]. We have also found an explicit relation [12] between the radius and the dimensionless amplitude $a_{0}$ in eq(11),

$$
a_{0}=1.73 \times 10^{-8} \frac{\left(10^{8} \mathrm{~cm}\right)^{2}}{R_{a}^{2}} \frac{10^{-5} \mathrm{eV}}{m_{a}}
$$

These explicit formulae are used for the evaluation of the dissipation energy of the axion stars in the magnetized conducting media.

\section{AXION STAR IN MAGNETIC FIELD}

We now proceed to explain that the axionic boson stars generate an electric field in an external magnetic field. It will turn out below that the field gives rise intriguing astrophysical phenomena. Thus it is important to understand the mechanism of producing the field. The point is that the axion couples [2] with the electromagnetic fields in the following way,

$$
L_{a \gamma \gamma}=c \alpha a \vec{E} \cdot \vec{B} / f_{P Q} \pi
$$

with $\alpha=1 / 137$, where $\vec{E}$ and $\vec{B}$ are electric and magnetic fields respectively. The value of $c$ depends on the axion models [14, 15]; typically it is the order of one.

It follows from this interaction that Gauss law is given by 


$$
\vec{\partial} \vec{E}=-c \alpha \vec{\partial} \cdot(a \vec{B}) / f_{P Q} \pi+\text { "matter" }
$$

where the last term "matter" denotes contributions from ordinary matters. The first term in the right hand side represents a contribution from the axion. Thus it turns out that the axion field has an electric charge density, $\rho_{a}=-c \alpha \vec{\partial} \cdot(a \vec{B}) / f_{P Q} \pi$, under the magnetic field $\vec{B}$ [11]. This charge density does not vanish only when the field configuration $a$ is not spatial uniform since $\vec{\partial} \vec{B}=0$. Thus the electric field, $E_{a}$ associated with this axion charge is produced such that $\vec{E}_{a}=-c \alpha a \vec{B} / f_{P Q} \pi$. Note that both of $\rho_{a}$ and $E_{a}$ oscillate with the frequency given by the mass of the axion in the case of the axion star since the field $a$ itself oscillates.

Obviously, this field induces an oscillating electric current $J_{m}=\sigma E_{a}$ in magnetized conducting media with electric conductivity $\sigma$. In addition to the current $J_{m}$ carried by ordinary matters, e.g. electrons, there appears an electric current, $J_{a}$, associated with the oscillating charge $\rho_{a}$ owing to the current conservation [1] $\left(\partial_{0} \rho_{a}-\vec{\partial} \vec{J}_{a}=0\right)$. This is given such that $\vec{J}_{a}=-c \alpha \partial_{t} a \vec{B} / f_{P Q} \pi$. It is important to note that this electric current is present even in nonconducting media like vacuum as far as the axion star is exposed to the magnetic field. On the other hand, current $J_{m}$ is present only in the magnetized conducting media.

Since $\partial_{t} a \sim m_{a} a$ in the case of the axion star, the ratio of $J_{m} / J_{a}$ is given by $\sigma / m_{a}$. Hence, $J_{a}$ is dominant in the media with $\sigma<10^{12} / s$, while $J_{m}$ is dominant in the media with $\sigma>10^{12} / s$; note that $10^{10} / s<m_{a}<10^{12} / s$ corresponding to the above constraint on $f_{P Q}$. Astrophysically, insides of neutron stars or white dwarfs [16], which are our concerns in this paper, possess electric conductivities large enough for $J_{m}$ to be dominant. On the other hand, envelopes or surfaces of the white dwarfs may have the small conductivities so as for $J_{a}$ to be dominant, although the envelopes of the neutron stars have still much large conductivities so that $J_{m}$ is dominant.

Now we discuss some implications of these electric currents. First we show that these electric currents, especially, $J_{m}$ yield thermal energies to the conducting media owing to Joule's heats. It implies that the axion stars dissipates their energies in the media. It also 
implies that the media increase their temperatures and as a result they radiate thermal photons more than before. In particular, it will be shown in next section that the dark white dwarfs become bright to be detectable, when they collide with the axion stars. Such white dwarfs have been supposed to be plausible candidates of MACHO and to be cooled sufficiently so that the optical detection of them are difficult without the heating by the collision.

Second we show that since the currents are oscillating, radiations are emitted. However, most of the radiations are absorbed inside of the conducting media. Thus observable radiations are only ones emitted around the surfaces of the media. In particular, the emission from the surfaces of the neutron stars and the white dwarfs is important to be discussed. But there exists a problem that we can not estimate precisely its luminosity since we do not have enough information about physical properties of the surfaces, e.g. electric conductivity, opacity, e.t.c. of the magnetized stars. Hence our estimation of the luminosity is necessarily ambiguous. However, it will be turned out that sufficient amount of the emission for observation is expected in the case of the neutron stars since electric conductivities of the surfaces ( so called envelopes ) are much larger than those of normal metals.

In order to see these expectations, first of all, we would like to calculate the dissipation energy of the axion star exposed to an magnetic field. The field is associated with the neutron star or the white dwarf [16]. We suppose such a situation that these magnetic stars collide with an axion star. As the radius of the axion star depends on $M_{a}$ and $m_{a}$, we need to treat two cases separately; the case of the radius of the axion star being larger than the radius $R$ of the medium, $R_{a}>R$ and the inverse case, $R_{a}<R$. Namely, the dissipation arises when the media are in inside of the axion star and it arises when the axion star is in inside of the media.

Denoting the average electric conductivity of the media by $\sigma$ and assuming the Ohm law, we find that the axion star dissipates an energy $W$ per unit time,

$$
W_{>}=4 \sigma \alpha^{2} c^{2} B^{2} a_{0}^{2} R^{3} / 3 \pi \quad \text { for } R_{a}>R
$$




$$
\begin{aligned}
& =5.5 c^{2} \times 10^{31} \mathrm{erg} / \mathrm{s} \frac{\sigma}{10^{22} / s} \frac{m_{a}^{6}}{\left(10^{-5} \mathrm{eV}\right)^{6}} \frac{M^{4}}{\left(10^{-14} M_{\odot}\right)^{4}} \frac{R^{3}}{\left(10^{9} \mathrm{~cm}\right)^{3}} \frac{B^{2}}{\left(10^{6} G\right)^{2}} \\
& =5.5 c^{2} \times 10^{38} \mathrm{erg} / \mathrm{s} \frac{\sigma}{10^{26} / s} \frac{m_{a}^{6}}{\left(10^{-5} \mathrm{eV}\right)^{6}} \frac{M^{4}}{\left(10^{-14} M_{\odot}\right)^{4}} \frac{R^{3}}{\left(10^{6} \mathrm{~cm}\right)^{3}} \frac{B^{2}}{\left(10^{12} G\right)^{2}} \\
W_{<} & =\sigma \alpha^{2} c^{2} B^{2} R_{a}^{3} a_{0}^{2} / 8 \pi \quad \text { for } R_{a}<R \\
& =4 c^{2} \times 10^{38} \mathrm{erg} / \mathrm{s} \frac{\sigma}{10^{22} / s} \frac{M}{10^{-12} M_{\odot}} \frac{B^{2}}{\left(10^{6} G\right)^{2}} \quad, \\
& =4 c^{2} \times 10^{54} \mathrm{erg} / \mathrm{s} \frac{\sigma}{10^{26} / s} \frac{M}{10^{-12} M_{\odot}} \frac{B^{2}}{\left(10^{12} G\right)^{2}}
\end{aligned}
$$

with $c \sim 1$. We have used the formulae eq(11), eq(12) and eq(13). Since the field $a$ oscillates [12,8 with a frequency given by the mass of the axion $m_{a}$, we have taken an average in time over the period, $m_{a}^{-1}$. When $R$ is much smaller than $R_{a}$, we have set, $\exp \left(-r / R_{a}\right)=1$ in eq(11) in the derivation of the formula eq(16); $r<R . W$ in the equations eq(17) and eq(20) is for the white dwarf [16] with $R \sim 10^{9} \mathrm{~cm}$ and $B \sim 10^{6} \mathrm{G}$, and $W$ in the equations eq(18) and eq(21) is for the neutron star [16] with $R \sim 10^{6} \mathrm{~cm}$ and $B \sim 10^{12} \mathrm{G}$, respectively. The values of $\sigma$ have been taken tentatively.

Note that $R_{a}=1.6 \times 10^{10} m_{5}^{-2} M_{14}^{-1} \mathrm{~cm}$. Hence formula $W_{<}$is applied to the white dwarf with $R=10^{9} \mathrm{~cm}$ only when $m_{5}^{2} M_{14}>10^{2}$, e.g. $m_{5}>10$ and $M_{14}>1$. On the other hand, $W_{<}$is applied to the neutron star with $R=10^{6} \mathrm{~cm}$ only when $m_{5}^{2} M_{14}>10^{4}$, e.g. $m_{5}>10$ and $M_{14}>1$

We comment that the formula may be applied to the conducting media where the Ohmic law holds even for oscillating electric fields with their frequencies $m_{a}=10^{10} \sim 10^{12} \mathrm{~Hz}$. In general the law holds in the media where electrons interact sufficiently many times in a period of $m_{a}^{-1}$ with each others or other charged particles and diffuse their energies acquired from the electric field. Actually the law holds in the white dwarfs and neutron stars of our concerns.

We would like to point out that although the electric field $\vec{E}_{a}=-c \alpha a \vec{B} / f_{P Q} \pi$ is much small owing to the large factor of $f_{P Q}$, the amount of the dissipation energy $W$ becomes large because $W$ is proportional to the volume $\left(R^{3}\right)$ of the media or the volume $\left(R_{a}^{3}\right)$ of the axion stars. 
Next we calculate the luminosity of the monochromatic radiations emitted around the surface of the magnetized media. They arise associated with the oscillation of the currents $J_{a}$ or $J_{m}$. Here we are only concerned with the case, $R_{a}>R$. This is because the radiations are emitted only around the surface of the media; it is possible when the media is in inside of the axion star.

We denote a depth of a region from the surface by $d$, in which radiations are emitted and can escape from the magnetized conducting media. We also denote an average electric conductivity in the region by $\sigma$. These values are not well known so that we take them as free parameters. Noting that only radiations from a semi-sphere facing observers can arrive at them, we calculate electromagnetic gauge potentials $A_{i}$ of the radiations with an appropriate gauge condition,

$$
\begin{aligned}
A_{i} & =\frac{1}{R_{0}} \int_{\text {surface }} J_{m}\left(t-R_{0}+\vec{x} \cdot \vec{n}\right) d^{3} x \\
& =\frac{c \alpha \sigma a_{0} B_{i}}{\pi R_{0}} \int_{\text {Surface }} \sin m_{a}\left(t-R_{0}+\vec{x} \cdot \vec{n}\right) d^{3} x \\
& =\frac{2 c \alpha \sigma a_{0} B_{i} R}{R_{0} m_{a}^{2}}\left(m_{a} d \cos m_{a}\left(t-R_{0}\right)-2 \cos m_{a}\left(t-R_{0}+R-d / 2\right) \sin \left(m_{a} d / 2\right)\right)
\end{aligned}
$$

where we have integrated it over the region around the surface with the depth $d \ll R$. $R_{0}$ is the distance between the observer and the media $\left(R_{0} \gg R\right)$. Here we have used the current $J_{m}=\sigma E_{a}$ with the field $a$ in the approximate formula eq(11) with setting $\exp \left(-r / R_{a}\right)=1$; the media is involved fully in the axion star so that $r / R_{a} \ll 1$. On the other hand, the current $J_{a}$ should be used for $\sigma \leq m_{a}$, in which case $\sigma$ should be replaced with $m_{a}$ in the above formula. Using the gauge potentials, we evaluate the luminosity of the monochromatic radiations with the frequency of $m_{a}$,

$$
L=\frac{8}{3}\left(\frac{\sigma}{m_{a}}\right)^{2} c^{2} a_{0}^{2} B^{2} R^{2} K^{2}
$$

where we have taken an average both in time and the direction of the magnetic field. $K^{2}$ is given such that

$$
K^{2}=\left(m_{a}^{2} d^{2}+4 \sin ^{2}\left(m_{a} d / 2\right)-4 m_{a} d \cos \left(m_{a} R\right) \sin \left(m_{a} d / 2\right)\right) / 2
$$




$$
\begin{aligned}
& \cong m_{a}^{2} d^{2} / 2 \quad \text { for } m_{a} d \gg 1 \\
& \cong m_{a}^{2} d^{2}\left(1-\cos \left(m_{a} R\right)\right) \quad \text { for } m_{a} d \ll 1
\end{aligned}
$$

In both limit $K^{2}$ is proportional to $m_{a}^{2} d^{2}$. Thus it turns out that $L$ is proportional to $\sigma^{2} d^{2}$ for $m_{a} \leq \sigma$, or to $m_{a}^{2} d^{2}$ for $\sigma \leq m_{a}$. We should note that the luminosity is proportional to the surface area $R^{2}$ of the magnetized stars; it is order of $\left(10^{6}\right)^{2} \mathrm{~cm}^{2}$ for neutron stars or $\left(10^{9}\right)^{2} \mathrm{~cm}^{2}$ for white dwarfs. Thus the quantity is enhanced even if a luminosity per unit area in the surface is quite weak. This is the point we wish to stress. As we have stated before, phenomena caused by the axion are too faint to be detected owing to small factor of

$m_{a} / f_{P Q}$. But in our case we have a large factor $R^{2} m_{a}^{2}$ of the order of, for example, $10^{18}$ in the case of the white dwarfs.

To evaluate numerically the value of $L$ we need to know the depth $d$ and the electric conductivity $\sigma$ around the surface. It seems that the quantities depend on each physical conditions of the surface of the magnetized stars, e.g. temperature, constituents e.t.c.. Thus it is difficult to estimate generally the luminosity. In latter sections we discuss it by assuming that the depth is given by a penetration depth of the radiations. Then the depth is written in terms of conductivity $\sigma$. With this simplification, only the conductivity around the surface remains as an ambiguous quantity.

\section{WHITE DWARF}

White dwarfs [16] are stars in the final stage of their lives with intermediate masses, $1 M_{\odot} \sim 8 M_{\odot}$. As is well known, they are composed of $\mathrm{C}$ or $\mathrm{O}$ with atmosphere of $\mathrm{H}$ or $\mathrm{He}$, and their mass ( radius ) is typically given by $0.5 M_{\odot}\left(10^{9} \mathrm{~cm}\right)$. The pressure in the white dwarfs is dominated by pressure of degenerate electrons whose density is much larger than normal metals. On the other hand, the internal energy is dominantly given by kinetic and potential energies of nuclei such as $\mathrm{C}$ and $\mathrm{O}$. Then, radiations from them reduce their internal energies stored inside. As a result their temperatures decrease and their luminosities become small with time because they never generate nuclear energies. Hence 
old white dwarfs are expected to be so dark that it is difficult to observe them. This fact leads to the natural expectation that the white dwarfs in the halo are candidates of MACHO detected with a gravitational microlense effect. Actually some of cooling models [17] of the white dwarfs support this expectation, although this point is still controversial. There are several arguments against this possibility of MACHO being the white dwarf [18].

Here we assume that MACHO is just a dark white dwarf with sufficiently low temperature whose population is $2 \times 10^{11} M_{\odot} / 0.5 M_{\odot} \sim 4 \times 10^{11}$; note that total mass of the halo is about $4 \times 10^{11} M_{\odot}$, half of which is expected to be the mass of the white dwarfs. We show that the dark white dwarfs become rebright with the collision of the axion stars. We also calculate the rate of the collision in the halo. As a result we find that the number of the white dwarfs in the halo increases discontinuously with luminosities below a certain luminosity ( $\left.10^{-5.5} L_{\odot} \sim 10^{-7} L_{\odot}\right)$ gained by the white dwarfs with the collision.

These white dwarfs may have strong magnetic fields typically such as $10^{6} \mathrm{G}$. Then when they collide with the axion stars, the axion field generates currents $J_{a}$ and $J_{m}$ in the white dwarfs. Consequently, thermal energies are produced with the dissipation of the axion field energy. The energies $\left(\propto \sigma J^{2}\right)$ are expected to be large owing to large electric conductivity in the white dwarfs. In particular, inside of sufficiently cooled white dwarfs is crystallized just like solid metals and reaches a stage of a fast Debye cooling [16]. It means that the old white dwarf has been sufficiently cooled [17] so that their core temperature is much lower than $10^{4} \mathrm{~K}$, for example. In such a case, the conductivity of electrons have been found [19] theoretically to be large; $\sigma \sim 10^{26}\left(T / 10^{3} \mathrm{~K}\right) / \mathrm{sec}$ at density $\sim 10^{7} \mathrm{~g} / \mathrm{cm}^{3}$ where $T$ represent a core temperature inside of the white dwarf.

Then, colliding with the axion star, the white dwarf gains the dissipation energy per unit time as follows,

$$
\begin{aligned}
& W_{>}=5.5 c^{2} \times 10^{35} \mathrm{erg} / \mathrm{s} \frac{\sigma}{10^{26} / s} \frac{m_{a}^{6}}{\left(10^{-5} \mathrm{eV}\right)^{6}} \frac{M_{a}^{4}}{\left(10^{-14} M_{\odot}\right)^{4}} \frac{R^{3}}{\left(10^{9} \mathrm{~cm}\right)^{3}} \frac{B^{2}}{\left(10^{6} G\right)^{2}} \quad \text { for } R_{a}>R \\
& W_{<}=4 c^{2} \times 10^{42} \mathrm{erg} / \mathrm{s} \frac{\sigma}{10^{26} / \mathrm{s}} \frac{M}{10^{-12} M_{\odot}} \frac{B^{2}}{\left(10^{6} G\right)^{2}} \quad \text { for } R_{a}<R
\end{aligned}
$$

where we have taken the above value of the conductivity. Noting that a constant $c$ is order 
of 1 , we found that the white dwarf gains so much thermal energy when it goes through the axion star.

However, the maximal energy the axion star can deposit per unit time is the energy possessed by a part of the axion star which the white dwarf sweeps per unit time, when the dwarf is smaller than the axion star $\left(R_{a}>R\right)$. This is owing to the energy conservation. As relative velocity, $v$, between the white dwarf and the axion star in the halo is approximately given by $10^{-3} \times$ light velocity, the energy stored in the part can be estimated such as $3 R^{2} v M / 4 R_{a}^{3} \sim 10^{35} \mathrm{erg} / \mathrm{s}\left(M_{a} / 10^{-14} M_{\odot}\right)^{4}\left(m_{a} / 10^{-5} \mathrm{eV}\right)^{6}$. This is smaller than $W_{>}$estimated naively. Therefore, real amount of the energy the white dwarf can gain is at most given by $W_{\text {real }} \sim 10^{35} \mathrm{erg} / \mathrm{s}\left(M_{a} / 10^{-14} M_{\odot}\right)^{4}\left(m_{a} / 10^{-5} \mathrm{eV}\right)^{6}$. The gain of the energy continues until the white dwarf passes through the axion star. Thus total energy gained by the white dwarf ( or dissipated by the axion star ) is $2 R_{a} / v \times W_{\text {real }} \sim 10^{38} \mathrm{erg}\left(M_{a} / 10^{-14} M_{\odot}\right)^{3}\left(m_{a} / 10^{-5} \mathrm{eV}\right)^{4}$ in the case of the radius of the axion star being larger than that of the white dwarf. This is the energy gained by the white dwarf when it passes the axion star without trapping the star. If the white dwarf traps the axion star, the white dwarf may gain larger amount of the energy. Maximally all of the mass $M_{a} \simeq 1.8 \times 10^{40} \mathrm{erg}\left(M_{a} / 10^{-14} M_{\odot}\right)$ can be transformed to the thermal energy.

On the other hand, when the dwarf is larger than the axion star, it is obvious from the formula eq(30) that the whole energy of the axion star, $M_{a} \simeq 1.8 \times 10^{42} \operatorname{erg}\left(M_{a} / 10^{-12} M_{\odot}\right)$ is dissipated within one second. Namely such a axion star evaporates soon after it enters the white dwarf.

Suppose that the white dwarf with mass $0.5 \times M_{\odot}$ and radius $10^{9} \mathrm{~cm}$ is in the stage of the Debye cooling. Then, their specific heat, $c_{v}$, per ion is given approximately by [16] $c_{v} \simeq 16 \pi^{4}\left(T / \theta_{D}\right)^{3} / 5$, where $\theta_{D}$ is the Debye temperature, typically being $10^{7} \mathrm{~K}$. Hence the injection of the energy, $10^{38} \mathrm{erg}\left(M / 10^{-14} M_{\odot}\right)^{3}\left(m_{a} / 10^{-5} \mathrm{eV}\right)^{4}$ ( or $\left.M_{a} \simeq 10^{42} \operatorname{erg}\left(M_{a} / 10^{-12} M_{\odot}\right)\right)$, increases the core temperature of the white dwarf to $\simeq 1.5 \times 10^{4} \mathrm{~K}\left(M / 10^{-14} M_{\odot}\right)^{3 / 4}\left(m_{a} / 10^{-5} \mathrm{eV}\right)\left(\right.$ or $\left.\simeq 1.5 \times 10^{5} \mathrm{~K}\left(M_{a} / 10^{-12} M_{\odot}\right)^{1 / 4}\right)$ when the initial temperature is much less than these ones. In order to evaluate the luminosity of 
the dwarf, we need to know the surface temperature. Generally, the surface temperature is much lower than the core temperature. It depends on opacity of the atmosphere of the white dwarf. Recently it has been shown [20] that the white dwarf with atmosphere of helium has much lower opacity at surface temperature $\left(<6 \times 10^{3} \mathrm{~K}\right)$ than ones estimated previously. Such a white dwarf cools more rapidly. According to the model [20] of the cooling, such white dwarfs of ages $\simeq 10^{10}$ years have been cooled with their core temperature $\simeq 2.3 \times 10^{5}$ $\mathrm{K}$ and with corresponding surface temperature $2000 \mathrm{~K}$; its luminosity is $10^{-5.6} L_{\odot}$ which is less than the minimum luminosity $\sim 10^{-4.8} L_{\odot}$ of a white dwarf observed at present. Thus white dwarfs with their ages older than $10^{10}$ years must be cooler than this one. It is expected that ages of the halo white dwarf population is around $1.2 \times 10^{10}$ years. Hence if the axion star with mass larger than $10^{-12} M_{\odot}\left(10^{-9} M_{\odot}\right)$ collides with the white dwarf whose radius is larger than that of the axion star, then the core temperature reaches more than $\simeq 1.5 \times 10^{5} \mathrm{~K}\left(\simeq 8.4 \times 10^{5} \mathrm{~K}\right)$ and its luminosity does more than $L \simeq 10^{-6} L_{\odot}\left(\simeq 10^{-4.5} L_{\odot}\right.$ ) according to the cooling model; here we have simply extrapolated its result to the case of lower luminosity than that $\left(\sim 10^{-5.6} L_{\odot}\right)$ addressed in the model. It takes about $1.4 \times 10^{7}$ years $\left(5 \times 10^{8}\right.$ years $)$ for the dwarf to loose the energy injected.

Now we proceed to estimate the rate of the collision between the white dwarf and the axion star in the halo. Especially, we are concerned with the event rate observed in a solid angle, $5^{\circ} \times 5^{\circ}$, for example. We assume that as indicated by the recent observations of gravitational microlensing, the half of the halo is composed of the white dwarfs with mass $M=0.5 \times M_{\odot}$ and radius $R=10^{9} \mathrm{~cm}$. The other half is assumed to be composed of the axion stars. Total mass of the halo is supposed to be $\sim 4 \times 10^{11} M_{\odot}$. Furthermore, the distribution 21] of the halo is taken such that its density $\propto\left(r^{2}+3 R_{c}^{2}\right) /\left(r^{2}+R_{c}^{2}\right)^{2}$ with $R_{c}=4 \mathrm{kpc}$ where $r$ denotes a radial coordinate with the origin being the center of the galaxy ( the final result does not depend practically on the value of $R_{c}=2 \sim 8 \mathrm{kpc}$ ). Then it is easy to evaluate the event rate of the collisions,

$$
0.5 \text { per year } \times \frac{\left(10^{-14} M_{\odot}\right)^{3}}{M_{a}^{3}} \frac{\left(10^{-5} \mathrm{eV}\right)^{4}}{m_{a}^{4}} \frac{\Omega}{5^{\circ} \times 5^{\circ}} \quad \text { for } R<R_{a}
$$




$$
5 \times 10^{-5} \text { per year } \times \frac{10^{-12} M_{\odot}}{M_{a}} \frac{\Omega}{5^{\circ} \times 5^{\circ}} \quad \text { for } R>R_{a}
$$

where $\Omega$ is a solid angle. We have taken into account the fact that the earth is located at about $8 \mathrm{kpc}$ from the center of our galaxy, simply by counting the number of the collisions arising in the region from $r=8 \mathrm{kpc}$ to $50 \mathrm{kpc}$. Here we have assumed that the collisions take place with their cross section simply given by $\pi\left(10^{10} \mathrm{~cm}\right)^{2}\left(10^{-14} M_{\odot} / M_{a}\right)^{2} m_{5}^{-4}$ for $R<R_{a}$ and $\pi\left(10^{9} \mathrm{~cm}\right)^{2}$ for $R>R_{a}$, respectively. Namely the cross section is geometrical one of the axion star or the white dwarf. But we expect that actual cross section is much larger than this one; a tidal force of the white dwarf may decrease kinetic energy of the axion star by tearing the star.

We also calculate the rate of the collision in the neighborhood of the earth. In particular, we with to see how many the collisions occur within a volume $(1 \mathrm{Kpc})^{3}$ in a year around the earth. This is because since the luminosity of the white dwarfs expected in the collisions is much small, the dwarfs need to be located near the earth in order to be detected. We assume that local density of the halo [3] is given by $0.5 \times 10^{-24} \mathrm{~g} \mathrm{~cm}^{-3}$, half of which is composed of the white dwarfs and the other half is composed of the axion stars. Then it is easy to find that the rate of the collisions is given by $\sim 0.06 M_{14}^{-3} m_{5}^{-4}$ per year for $R<R_{a}$ and $\sim 6 \times 10^{-6} M_{12}^{-1}$ per year for $R>R_{a}$, respectively, where we used the relative velocity being equal to $3 \times 10^{7} \mathrm{~cm} / \mathrm{sec}$. It seems that it is difficult to detect the collisions. Real population of the white dwarfs and the axion stars in the halo may be smallar than one we have assumed in the analysis. Then the collision rate is smaller than the above value and it is more difficult to detect the collision with observation of monochromatic radiations discussed below. However, if we take into account the fact that actual collision cross section must be much larger than the geometrical one of the axion star due to gravitational attraction, the rate will increase. To see it we need to analyze numerically the collision in detail.

Here we with to discuss how many white dwarfs heated with the collision are present in a region around the earth whose volume is assumed to be $(1 \mathrm{Kpc})^{3}$. For the purpose we note that the white dwarfs heated with the collision loose the energies injected, taking many 
years. Hence even if the collision rate is small, the number of such white dwarfs increases with time and saturates at a balance point between the decay and the production. For example it takes $1.4 \times 10^{7}$ years $\left(5 \times 10^{8}\right.$ years $)$ for a white dwarf with luminosity $10^{-6} L_{\odot}$ $\left(10^{-4.5} L_{\odot}\right)$ to loose the energy which is injected by an axion star with $M=10^{-12} M_{\odot}$ ( $\left.M=10^{-9} M_{\odot}\right)$. On the other hand, the rate of the collision producing the dwarf with the luminosity is given by $\simeq 6 \times 10^{-6}$ per year $\left(\simeq 6 \times 10^{-9}\right.$ per year $)$ in the region with volume $(1 \mathrm{Kpc})^{3}$. Thus it turns out that there present $84(3)$ such white dwarfs in the region. The number of such white dwarfs becomes larger as their luminosities gained with the collision are smaller. For example, with the collision of the axion star $M=10^{-11} M_{\odot}$, a white dwarf gains a luminosity $10^{-5.5} L_{\odot}$ and looses its energy in $3.7 \times 10^{7}$ years. On the other hand, the production rate of the dwarfs is $6 \times 10^{-7}$ per year. Thus there present approximately 22 such dwarfs. Accordingly, we understand that as the mass of the axion stars is smaller, the energy gained by the white dwarfs is smaller and resultant their luminosity is smaller, but the number of such dwarfs is larger.

Although we do not know theoretically the masses of the axion stars, observations of dark white dwarfs enable us to determine the mass of the axion stars. Namely if there is a threshold luminosity e.g. $10^{-5.5} L_{\odot}$ below which the number of the white dwarfs increases discontinuously, it implies that such white dwarfs with the threshold luminosity are produced with the collisions of the axion stars with a corresponding mass e.g. $10^{-11} M_{\odot}$. In this discussion, we have assumed that old white dwarfs dominant in the halo have been cooled sufficiently and their core temperature is quite low (e.g. less than $10^{4} \mathrm{~K}$ ).

Until now, we have discussed the thermal radiations caused by the axion star which dissipates its energy and makes the white dwarf warmer than before.

We proceed to discuss the monochromatic radiations generated by the oscillating electric currents. The radiations are ones emitted only around the surface of the white dwarf. Otherwise, the radiations are absorbed inside of the white dwarf. There are difficult problems to estimate amount of such radiations because the physical parameters ( conductivity, opacity, e.t.c. ) around the surface of the cooled white dwarfs have not yet been known so well 
[17,20]. Thus our estimation is inevitably ambiguous. However, with an assumption that the depth $d$ of the region in which the radiations are generated and can go out of the white dwarf, is given by a penetration depth of the radiations, we can obtain the amount of the radiations definitely with one ambiguous parameter, the penetration depth, which is written in terms of the electric conductivity and the frequency of the radiations.

With use of eqs(25) and (26), we find the luminosity of the radiations,

$$
\begin{aligned}
L & \sim 10^{21} \mathrm{erg} / \mathrm{s} B_{6}^{2} R_{9}^{2} \sigma_{20} m_{5}^{5} M_{14}^{4} c^{2} \quad \text { for } m_{a} \ll \sigma \\
& \sim 10^{21} \mathrm{erg} / \mathrm{s} B_{6}^{2} R_{9}^{2} m_{5}^{8} M_{14}^{4} c^{2} / \sigma_{5}^{2} \text { for } m_{a} \gg \sigma
\end{aligned}
$$

where

$$
B_{6}=\frac{B}{10^{6} \mathrm{G}}, \quad R_{9}=\frac{R}{10^{9} \mathrm{~cm}}, \quad \sigma_{20}=\frac{\sigma}{10^{20} / \mathrm{s}}, \quad \sigma_{5}=\frac{\sigma}{10^{5} / \mathrm{s}} .
$$

Here we have only addressed the case that the radius of the white dwarf is smaller than $R_{a}$. We have used the penetration depth $d=1 / 2 \pi \sigma$ for $m_{a} \gg \sigma$, and $d=\sqrt{1 / 2 \pi \sigma m_{a}}$ for $m_{a} \ll \sigma$, respectively. In both case we have simply assumed that values of both dielectric constant and magnetic permeability are the same as those of the vacuum. Numerically, $d \sim 10^{5} \mathrm{~cm} / \sigma_{5}$ for $m_{a} \gg \sigma$ and $d \sim 10^{-5} \mathrm{~cm}\left(\sigma_{20} m_{5}\right)^{-0.5}$ for $m_{a} \ll \sigma$. We should note that as a source of the radiations, matter current $J_{m}$ carried by electrons is dominant for $m_{a} \ll \sigma$, while axion current $J_{a}$ is dominant for $m_{a} \gg \sigma$. In each case we have represented luminosity in eq(33) and eq(34), respectively.

These monochromatic radiations are emitted only during the collision with the axion star. This is because after the white dwarf passes the axion star, there are not any oscillating currents around its surface; the currents arises only when the dwarf is passing the inside of the axion star. Therefore, the period of the emission continueing is given by $2 R_{a} / v \sim$ $3.2 \times 10^{10} \mathrm{~cm} m_{5}^{-2} M_{14}^{-1} /\left(3 \times 10^{7} \mathrm{~cm} / \mathrm{s}\right) \sim 10^{3} \mathrm{sec}$ for $m_{5}^{2} M_{14}=1$.

It seems that the luminosity is small for the observation of these radiations. But it depends heavily on the mass of the axion especially in the case of $m_{a} \gg \sigma$. For example if $m_{a}=10^{-4.5} \mathrm{eV}$ where $R \sim R_{a}$, then $L \sim 10^{25} \mathrm{erg} / \mathrm{s}$ in the case of $m_{a} \gg \sigma$, eq(34). 
This may be large enough for the observation. Furthermore, we point out that there are huge ambiguities in the evaluation of the luminosity as we have mentioned before. Thus we can not determine definitely whether the luminosity of the radiations is large enough for observation, or not. Although there exist some parameter ranges for the radiations being observable, it seems difficult to observe the radiations caused with the collision unless the luminosity is so large for the radiation from the distance $10 \mathrm{Kpc}$ to be detectable. Note that the rate of the collision is given approximately by $60 M_{14}^{-3} m_{5}^{-4}$ per year per $(10 \mathrm{Kpc})^{3}$.

In the above discussion we have assumed that the axion star does not receive any gravitational effects from the white dwarf in the collision. However, actually it receives strong gravitational effects from the white dwarf. This is because the mass of the white dwarf is much bigger than that of the axion star. So we need to take into account the effects in order to see whether or not some of the assumptions are changed, in particular, the number of the axion stars which was estimated naively under an assumption of the stars not decaying within the age of the Universe.

First of all, we examine whether or not the axion star decays by a tidal force when the white dwarf passes near it. We suppose that the axion star decays if an energy difference caused by the tidal force between different parts of the axion star is larger than the binding energy of the axion star. In particular, we wish to estimate how many the axion stars survive without decaying in the present Universe whose age is approximately $10^{10}$ years.

Suppose that an axion star with mass $10^{-14} M_{\odot}$ is composed of two parts which are bounded gravitationally with each other; the distance between the two parts is assumed to be $R_{a}$. When a white dwarf passes the axion star, each part receives different gravitational force owing to the difference of their distances from the white dwarf. In order to examine whether or not this tidal force tears the axion star, we compare an energy difference between an gravitational energy received by a part of the axion star from the white dwarf and an energy received by the other part of the axion star, with the binding energy $G M_{a}^{2} / R_{a}$ of the axion star. It is reasonable to think that if the energy difference is larger than the binding energy, the tidal force tears the axion star. The energy difference is dependent on the impact 
parameter of the collision between the axion star and the white dwarf. Assuming a relative velocity being $10^{-3} \times$ light velocity, we find with rough estimation that the axion star decays with the tidal force when they approach within $10^{15} \mathrm{~cm}$ each other. Since the number of the axion stars in the halo is given by $\sim 2 \times 10^{11} M_{\odot} / 10^{-14} M_{\odot}=2 \times 10^{25}$, and the number of the white dwarfs is $\sim 2 \times 10^{11} M_{\odot} / 0.5 \times M_{\odot}=4 \times 10^{11}$, the number of the collisions leading to the decay of the axion stars is at most the order of $10^{23}$ within the age of the Universe. Therefore, it turns out that almost of all axion stars ( more than 99 percent ) have survived against such collisions. Note that the axion stars with masses larger than $10^{-14} M_{\odot}$ can survive even more against the tidal force of the white dwarfs. This is because the collision cross section becomes smaller for such axion stars and so the decay rate is smaller.

Although we found that there still present quite large numbers of the axion stars in our halo, we do not know how actually direct collisions between the axion star and the white dwarf take place; a direct collision implies a collision with their closest distances being less than $R_{a} \sim 10^{10} \mathrm{~cm}$. Namely its cross section is given by $\pi\left(1.6 \times 10^{10} \mathrm{~cm}\left(10^{-14} M_{\odot} / M_{a}\right)\right)^{2}$, i.e. the geometrical cross section of the axion star itself. It seems that the gravitational force deforms strongly the configulation of the axion star, but the coherence of the axion field may holds and the generation of the electric currents still arises. Thus we expect the heat up of the cooled white dwarfs and the resultant emissions of the thermal radiations as well as the monochromatic radiations.

\section{NEUTRON STARS}

Now we proceed to discuss the influence on neutron stars of their collision with the axion stars. The neutron star [16] is highly dense nuclear matter composed of neutrons and protons, although number of the protons is much less than that of neutrons. Since their radius, $R_{n}$, is typically given by $10^{6} \mathrm{~cm}$, we are only concerned with the case of $R_{n}<$ $R_{a} \sim 10^{8} m_{5}^{-2} M_{12}^{-1} \mathrm{~cm}$. Since there are also highly dense electrons, conductivity is quite higher than that of the white dwarf. As is well known, strong magnetic field is present. 
Their strength is typically given by $10^{12} \mathrm{G}$. Hence, the amount of the energy dissipation of the axion star per unit volume in the neutron star is much larger than that in the white dwarf. But as we mentioned in previous section, the actual amount of the energy dissipation is restricted owing to the energy conservation. It is given by $3 R_{n}^{2} v M_{a} / 4 R_{a}^{3} \sim$ $10^{29} \mathrm{erg} / \mathrm{s}\left(M_{a} / 10^{-14} M_{\odot}\right)^{4}\left(m_{a} / 10^{-5} \mathrm{eV}\right)^{6}$. Total amount of the energy deposited after the collision is $10^{32} \operatorname{erg}\left(M_{a} / 10^{-14} M_{\odot}\right)^{3} m_{5}^{4}$. This is a small fraction of the thermal energy possessed by the neutron star. Therefore, the collision with the axion star does not affect significantly thermal contents in the neutron star, contrary to the case of the white dwarf.

Although the thermal energy does not change so much with the collision, detectable amount of radiations from the surface of the neutron star arises during the collision with the axion star. As we mentioned, the axion field of the axion star generates an oscillating electric field $E_{a}$ when it is under a magnetic field. Thus the oscillating current $J_{m}=\sigma E_{a}$ is induced around the surface, which generates obviously the radiations. In the case of the neutron stars the electric conductivity is so large [19] even at the surface that the luminosity of the radiations is large enough for them to be detectable.

Using the formula eq(25) and eq(26), we obtain the luminosity of the radiations,

$$
L \sim 10^{27} \mathrm{erg} / \mathrm{s} B_{12}^{2} R_{6}^{2} \sigma_{20} m_{5}^{5} M_{14}^{4}
$$

with

$$
B_{12}=\frac{B}{10^{12} G}, \quad R_{6}=\frac{R_{n}}{10^{6} \mathrm{~cm}}, \quad \sigma_{20}=\frac{\sigma}{10^{20} / \mathrm{s}}
$$

where we have used the above formulae eq(12) and eq(13) for expressing $a_{0}$ in terms of the mass $M_{a}$ of the axion star. We have assumed for convenience that the conductivity takes a value such as $10^{20} / \mathrm{s}$. It is reasonable to take such a value of $\sigma$ because the number density of electrons at the surface must be much larger than that of normal metals. Then since it is much larger than $m_{a}$, the depth $d$ is taken such as $d=\sqrt{1 / 2 \pi \sigma m_{a}}$ which is the penetration depth of the radiations. This luminosity is that of the monochromatic radiation with the frequency, $m_{a} / 2 \pi=2.4 \times 10^{9} m_{5} \mathrm{~Hz}$. 
The emission continues until the axion star passes through the neutron stars. It takes $2 R_{a} / v \sim 3.2 \times 10^{10} \mathrm{~cm} m_{5}^{-2} M_{14}^{-1} /\left(3 \times 10^{7} \mathrm{~cm} / \mathrm{s}\right) \sim 10^{3} \mathrm{sec} m_{5}^{-2} M_{14}^{-1}$, assuming that the velocity of the axion star is $3 \times 10^{7} \mathrm{~cm} / \mathrm{s}$, which is the typical velocity of matters composing the halo in our galaxy.

The monochromatic radiations emitted at a distance $D$ from the earth is detected at the earth with the following strength,

$$
\sim 1 \mathrm{Jy} B_{12}^{2} R_{6}^{2} \sigma_{20} m_{5}^{4} M_{14}^{4}\left(D_{k p c}\right)^{-2}
$$

with $D_{k p c}=D / 1 \mathrm{Kpc}$, where we have assumed that the frequency $\nu$ of the radiations is Doppler broadened with a characteristic width $\Delta \nu \sim 10^{-3} \nu$ owing to the velocity of the axion stars. Unit of Jy denotes Jansky; $1 \mathrm{Jy}=10^{-23} \mathrm{erg} \mathrm{cm}^{-2} \mathrm{sec}^{-1} \mathrm{~Hz}^{-1}$. It turns out that the strength of the radiations is large enough to be detectable although the duration $\sim 10^{3} \mathrm{sec}$ of emitting the radiations is very short.

In order to estimate how frequent the events of the collisions between the neutron star and the axion star occur in a neighborhood of the earth, for example, $D \leq 1 \mathrm{Kpc}$, we need to know number density of the neutron stars including old invisible ones in our galaxy. Assuming the number of the axion stars in the halo being given by $2 \times 10^{11} M_{\odot} / 10^{-14} M_{\odot} \sim 10^{25}$ and the their uniform distribution within a volume $\sim(50 \mathrm{Kpc})^{3}$, we find that the events in a volume $\sim(1 \mathrm{Kpc})^{3}$ occur with a rate of the order $\sim 10^{-9}$ per year when the number of the neutron stars in the volume is just one. Here the collision cross section is assumed to be given by geometrical one $\pi\left(10^{10}\right)^{2} \mathrm{~cm}^{2}$ of the axion star. Therefore it is impossible in practice to detect such phenomena unless the number density of the invisible old neutron stars in the neighborhood of the earth is much larger than one we expect, or the real collision cross section including the gravitational effect of the attraction is much larger than the geometrical one. 


\section{DISCUSSION}

We have shown that the axionic boson stars dissipate their energies in the magnetized conducting media such as the white dwarfs or the neutron stars. Among them, the old halo white dwarfs with sufficiently low core temperature $\left(<10^{3} \mathrm{~K} \sim 10^{4} \mathrm{~K}\right)$, possible candidates of MACHO, are heated in this mechanism and emit the thermal radiations. Their luminosities have been estimated to achieve more than a luminosity $10^{-6} L_{\odot}\left(10^{-7.1} L_{\odot}\right)$ of a white dwarf when $M_{a}$ is larger than $10^{-12} M_{\odot}\left(10^{-14} M_{\odot}\right)$; in the case of mass, $10^{-14} M_{\odot}$ we have assumed the axion stars being trapped to the white dwarf and dissipating their whole energy. These dwarfs loose their energies with the radiations and become dark with time, while new such dwarfs are produced with the collision of the axion stars. The masses of almost of all axion stars could be fixed to a certain value in a range of $10^{-14} M_{\odot} \sim 10^{-11} M_{\odot}$ when they are produced at QCD phase transition. Thus the core temperature or the luminosity of the dwarf heated with the collision must be almost the same as each other. Therefore, we expect that there exist a threshold luminosity below which the number of the halo white dwarfs increases discontinuously. The threshold is determined with the mass of the axion stars which collide and heat the dark dwarfs with sufficiently low temperature. In our estimation the threshold luminosity is given by $10^{-5.5} L_{\odot} \sim 10^{-7} L_{\odot}$ corresponding to the masses of the axion stars quoted above.

The threshold luminosity is quite small so that the collision need to occur near the earth for the detection of such white dwarfs. Thus we have also estimated the rate of the collision within a volume of $(1 \mathrm{Kpc})^{3}$ around the earth. With assumptions of both populations of the white dwarfs and the axion stars being given by half of the halo, the rate has been turned out to be $\sim 0.06 M_{14}^{-3} m_{5}^{-4}$ per year for $R<R_{a}\left(6 \times 10^{-6} M_{12}^{-1}\right.$ per year for $\left.R>R_{a}\right)$, when the relative velocity between the dwarf and the axion star being equal to $3 \times 10^{7} \mathrm{~cm} / \mathrm{sec}$; $R_{a}=1.6 \times 10^{10} m_{5}^{-2} M_{14}^{-1} \mathrm{~cm}$. This indicates that the detection of the phenomena like a nova caused with the collision would be difficult. But in the estimation we have not included the gravitational attraction. When we take into account the effect, the collision cross section 
will be quite larger than the naive geometrical one we have used in the estimation. So we may expect that the cross section will become large so that the actual rate of the collision is large for us to be able to observe the phenomena. On this point we need to simulate numerically the collision and to know how the collision occurs. In particular we with to know the collision cross section and also to know whether or not the axion star is teared by the tidal force of the white dwarf.

We have also shown that monochromatic radiations are emitted during the collision between the axion star and the magnetized conducting media. The radiations are produced around the surface of the media by oscillating current, $J_{m}=\sigma E_{a}$. Especially, strong radiations are expected from neutron stars since their electric conductivity $\sigma$ is still large even at their envelope. However, number density of the neutron stars in our galaxy may be much small for the rate of the collision to be fairly rare; $\sim 10^{-9} / \mathrm{Kpc}^{3}$ per year when their number density is given by $1 / \mathrm{Kpc}^{3}$. Thus it is difficult to observe the monochromatic radiations from the neutron stars.

On the other hand, although the collision with white dwarfs does not lead to strong radiations such as ones from the neutron stars, the number density of the dwarfs is supposed to be much larger than that of the neutron stars so that the rate of the collision is much larger than one in the case of the neutron stars. It is approximately given by $0.06 / \mathrm{Kpc}^{3}$ per year with use of $M_{a}=10^{-14} M_{\odot}$ and with use of the geometrical cross section $\left(=\pi R_{a}^{2}\right.$ ). Hence unless the luminosity of the radiations is large enough for them from the distance $10 \mathrm{Kpc}$, for example, to be detectable, the value is a little bit small for the observation. Furthermore, our assumption of the population of the white dwarfs and the axion stars is dubious. In general the population is smaller than one we have assumed. Then the collision rate is smaller than one in the above estimation. Hence real rate of the collision may be small enough so as for the collision to be undetectable. But taking account of gravitational attraction between the axion star and the dwarf, real cross section may be larger than the naive geometrical one. Thus the rate of the collision may be larger than one we have estimated. On this point we need to analyse numerically the way of the collision in detail. 
Finally, we mention that our estimation is ambiguous in a sense that we do not know many physical parameters associated with the phenomena, for instance, the precise values of both the mass and the population of the axion stars, the mass of the axion itself, physical properties of invisible old white dwarfs, actual cross section of the collision and how the axion star collides with the magnetic stars. Although there are many ambiguous points in the disscusion, it is important to note that the observation of the monochromatic radiations makes us determine precisely the mass of the axions and the observation of the threshold in luminosity function of the halo white dwarfs makes us determine the mass of the axion stars.

Theoretically, if we detect the monochromatic radiations which are emitted in a short period, they are good signal indicating that the collision between the axion star and the magnetized media actually occur. After the detection we expect to see a nova in the direction of the radiations; the nova is a white dwarf heated with the energy dissipation of the axion star in the old white dwarf.

A part of this work have been down when the author has visited the Theoretical Physics Group at LBNL. He would like to express his thank for useful discussions and comments to Professors J. Arafune and B. Hansen, and also for the hospitality in LBNL as well as in Tanashi KEK. 


\section{REFERENCES}

[1] R.D. Peccei and H.R. Quinn, Phys. Rev. Lett. 38, 1440 (1977),

S. Weinberg, Phys. Rev. Lett. 40, 223 (1978),

F. Wilczeck, Phys. Rev. Lett. 40, 279 (1978).

[2] J.E. Kim, Phys. Rep. 150, 1 (1987).

[3] E.W. Kolb and M.S. Turner, The Early Universe, Addison-Wesley, (1990).

[4] A. Iwazaki, Phys. Lett. B406, 304 (1997); Phys. Rev. Lett. 79, 2927 (1997); Phys. Rev. D56, 2437 (1997).

[5] C.J. Hogan and M.J. Ree, Phys. Lett. 205B, 228 (1988).

[6] E.W. Kolb and I.I. Tkachev, Phys. Rev. Lett. 71, 3051 (1993), Phys. Rev. D49, 5040 (1994).

[7] E.W. Kolb and I.I. Tkachev, Phys. Rev. D50, 769 (1994).

[8] E. Seidel and W.M. Suen, Phys. Rev. Lett. 66, 1659 (1991).

[9] E.W. Kolb and I.I. Tkachev, astro-ph/9510043,

I.I. Tkachev, Phys. Lett. B191, 41 (1987).

[10] C. Alcock et al., Phys. Rev. Lett. 74, 2867 (1995).

[11] P. Sikivie, Phys. Lett. B137, 353 (1984).

[12] A. Iwazaki, hep-ph/9804369.

[13] P. Jetzer, Phys. Rep. 220 (1992),

T.D. Lee and Y. Pang, Phys. Rep.

[14] A.R. Zhitnitsky, Sov. J. Nucl. Phys. 31, 260 (1980),

M.D. Dine, W. Fischler and M. Srednicki, Phys. Lett. B104, 199 (1981). 
[15] J. E. Kim, Phys. Rev. Lett. 43, 103 (1979),

M.A. Shifman, A.I. Vainshtein and V.I. Zakharov, Nucl. Phys. B166, 493 (1980).

[16] S.L. Shapiro and S.A. Teukolsky, Black Holes, White Dwarfs, and Neutron Stars, A Wiley-Interscience Publication, (1983).

[17] F. D'Antona and I. Mazzitelli, Annu. Rev. Astron. Astrophys. 28, 139 (1990), D.S. Graff, G. Laughlin, and K. Freese, Astrophys. J. 499, 7 (1998).

[18] R. Canal, J. Isern, and P. Ruiz-Lapuente, astro-ph 9708178.

[19] D.A. Baiko and D.G. Yakovlev, Astron. Lett. 72, 702 (1995), astro-ph/9604164.

[20] B.M.S. Hansen, Nature. 394860 (1998).

[21] C. Alcock et al., Astrophys. J. 449, 28 (1995). 